\title{
A master-slave haptic system for neurosurgery
}

\author{
Vanni Zanotto ${ }^{\mathrm{a}, *}$, Paolo Boscariol ${ }^{\mathrm{a}}$, Alessandro Gasparetto ${ }^{\mathrm{a}}$, Albano Lanzutti $^{\mathrm{a}}$, Renato Vidoni ${ }^{\mathrm{a}}$, \\ Nicola Di Lorenzo ${ }^{\mathrm{b}}$, Pasquale Gallina ${ }^{\mathrm{b}}$, Aldo Dalla Via ${ }^{\mathrm{c}}$ and Aldo Rossi ${ }^{\mathrm{c}}$ \\ ${ }^{\mathrm{a}}$ DIEGM Università di Udine, Udine, Italy \\ ${ }^{\mathrm{b}}$ Dipartimento di Neurochirurgia, Università di Firenze, Firenze, Italy \\ ${ }^{\mathrm{c}}$ DIMEG Università di Padova, Padova, Italy
}

\begin{abstract}
In recent years, new surgical tools have been designed to improve treatment results and lower patient trauma. Nevertheless, the dexterity and accuracy required for the positioning of new tools are often unreachable, if surgeons are not assisted by suitable systems. Significant advantages are derived from the introduction of computer and robot technologies. For that reason, the interaction between robotic systems and surgeons today is producing new interest worldwide both in medical and engineering fields. In particular, medical robotics has found fruitful ground in neurosurgical applications, since the high functional density of the central nervous system requires strict accuracy constraints on tool positioning. As a matter of fact, the major benefits of robots, such as precision, accuracy and repeatability, make them ideal as neurosurgeons' assistants. This paper presents a masterslave haptic robotic system for minimally invasive neurosurgery, which can aid surgeons in performing safer and more accurate stereotactic neurosurgical treatments. The design of the proposed system is based on LANS (Linear Actuator for NeuroSurgery), which has been developed by our Research Group. Experimental test aimed at showing the added value of the DAANS system over its predecessor, the effectiveness of conformational caps and of the added rotational degree of freedom are scheduled for the upcoming months.
\end{abstract}

Keywords: Neurosurgery, haptic systems, minimally-invasive neurosurgery, PRS, DAANS

\section{Introduction}

The initial experimentation of robotic systems in surgery was undertaken during the early 1980 s $[5,12$, 13 , and it basically consisted of adapting the industrial robot technologies already in existence. In the last decade, there has been a growing awareness, within the medical community, of the benefits offered by using robots in various surgical tasks.

Therefore, the interaction between robotic systems and surgeons is causing a new widespread interest

*Corresponding author: Vanni Zanotto, DIEGM Università di Udine, via delle Scienze 208, 33100, Udine, Italy. E-mail: vanni. zanotto@uniud.it. in the fields of medicine and robotics. Currently, this co-operation between engineers and physicians leads to considerable robotic innovations. Surgeons can make use of new capabilities to perform less invasive and more accurate surgical interventions, reducing the post-operative stay and improving clinical outcomes.

In particular, traditional surgery involves making large incisions to access the part of a patient's body that needs to be operated on. Minimally Invasive Surgery (MIS), on the other hand, is a cost-effective alternative to open surgery. Basically, the same operations are performed using instruments designed to enter the body cavity through several tiny incisions, rather than a single large one. By eliminating large incisions, trauma to 
the body, post-operative pain, and the length of hospital stay are significantly reduced.

However, new problems connected to the use of robots in surgery have arisen, since there is no direct contact with the patient. For this reason, it is necessary to develop suitable tactile sensors to provide surgeons with the perception of directly operating on the patient. Such a result can be achieved by using force feedback systems, in which the force applied to patient's tissue is fed back to a robotic device (haptic master) directly operated by the surgeon.

Haidegger and colleagues in [9] categorize surgical robots based on their different roles during surgical treatment. Passive robots only serve as a tool-holding device once directed to the desired position. Semiactive devices perform the operation under direct human control. Active devices are under computer control and automatically perform certain interventions.

Moreover, the surgical robot can have different levels of autonomy. To be specific, systems that are able to perform fully automated procedures are called autonomous. On the other hand, when the surgeon completely controls every single motion of the robot, this is called a tele-operated system or a master-slave system.

Medical robotics has found fruitful ground especially in neurosurgical applications, owing to the accuracy required by the high functional density of the central nervous system $[2,12,16,19]$.

The first documented application of medical robotics in neurosurgery was in the 1985 when Khow [12] used a commercial robot PUMA 200 by Unimation as positioning device for a biopsy instrument. In 1991 Drake [4] used the same robot as retraction device in the surgical management of thalamic astrocytomas. Despite their very innovative application, both systems lacked the proper safety features needed for widespread acceptance into neurosurgery. In 1987, Benabid [1] experimented with an early precursor to the robot marketed as Neuromate (Renishaw, UK). Neuromate uses preoperative image data to assist with surgical planning and a passive robotic arm to perform the procedure [13].

Neuromate (Fig. 1) is a five axis open-chain robot. It can be used both in frame-based and in frame-less neurosurgical operations. In the frame-based operations, the patient's head is held by means of a mechanical frame which works as a precise reference for the tool positioning. In the frame-less operations, on the other hand, the are no mechanical structures on the patient's

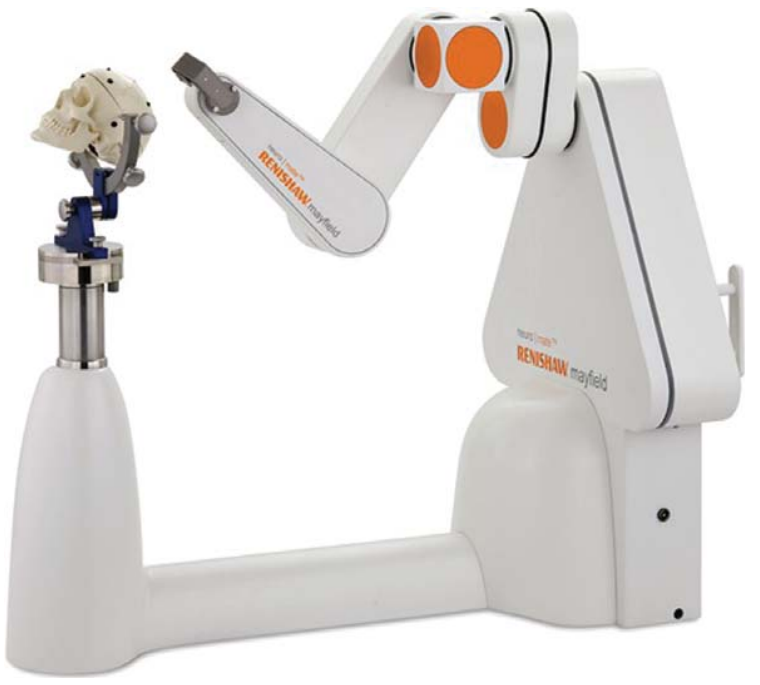

Fig. 1. Neuromate ${ }^{\circledR}$ (courtesy of Renishaw Mayfield, UK).

head but some special markers allow a vision system to estimate the correct head position.

Neurobot $[11,14]$ is a manipulator made of by two robots: the first one is a gantry robot which works as gross-motion positioner for the second one, a four axis robot which moves the surgical tool during the operations. The decoupling of the two robot motion increases the safety of the operation, since it reduces the mechanical components moving close to the patient. Moreover, since the number of actuators is reduced, the system features a higher level of accuracy. Neurobot is employed principally as an actuator for neurosurgical endoscopes.

Evolution 1 [21] is another example of robotized endoscope. It is made of a five axis robot which moves a parallel robot, on which a linear actuator moving the surgical tools is fixed. The first robot acts as gross-motions positioner, while the last allow a fine movement.

Liu and colleagues in [20] present a master-slave robotic system, named NeuroMaster, developed at the Robotics Institute of Beihang University. NeuroMaster is a five axis serial robot. The first axis is a prismatic joint while the four remaining joints are revolute. The prismatic joint allows a high accuracy in the vertical positioning of the tool.

Starting from the consideration that the accuracy in the tool positioning depends on the number of the robot axis, Modrak and colleagues in [15] present a new structure of neurosurgical manipulators with a reduced 
number of links. In particular they designed a surgical robot with only three revolute joints and one prismatic joint. This robot is passive and it works only as tools holder before the surgical operation.

These neurosurgical robots relied on preoperative images to determine robotic positioning. As a result, surgeons could not dynamically monitor needle placement under image-guidance and were blind to changes such as brain shift. To satisfy the need for a real-time, image-guided system, the Minerva system was developed (University of Lausanne, Lausanne, Switzerland). The robot arm is placed inside a computed tomography (CT) scanner, thus allowing surgeons to monitor the operation in real-time and make appropriate adjustments to the trajectory as needed [3, 13]. Despite considerable engineering challenges, the design and construction of magnetic resonance (MR) compatible robotic systems soon followed. MR compatibility ensures that the robot produces minimal MR imaging (MRI) artifact and that the operation of the robot is not disturbed by the electromagnetic fields.

Louw in [8] present another MRI compatible robot named NeuroArm, made of two eight axis serial manipulators. The redundancy allows to move the tool linearly with a high accuracy.

An up-to-date overview of the most significant researches conducted in this field can be found in [13] and in the references there in.

Today's robot projects focus on three major areas of improvement [9]:

- increasing the overall accuracy of the classical stereotactic systems

- increasing the added-value of the equipment

- enhancing the capabilities of the surgeon.

The present work falls into the second area. The system is based on a modified Neuromate robot in such a way that it can easily and accurately move a miniaturized X-ray source. Even if the system would be able to perform fully automated procedures, the surgeon controls every single motion of the robot in a masterslave, tele-operated manner, with force-feedback from the surgical tool. This solution permits highly safe conditions during treatment.

This paper is organized as follows. The next section provides a brief introduction of MIS by means of a miniaturized X-ray source and a description of the first neurosurgical actuator developed by our Research Group.
Afterwards, an improved surgical treatment is discussed. Finally, the new robotic actuator, named DAANS (Double Action Actuator for NeuroSurgery), which is able to meet the actuation requirements rising from the new treatment, will be presented.

\section{The photon radiosurgery system}

The PRS (Photon Radiosurgery System by Carl Zeiss $\left.{ }^{\circledR}\right)$ is a miniaturized radiosurgical device for Interstitial Radiation (IR) [6, 7, 17]. The device emits low energy X-rays from the tip of a cylindrical probe (Fig. 2a).

From the point of view of radiobiology, the IR with PRS aims at delivering a necrotizing dose of radiation in the tumor volume, minimizing the dose in the surrounding tissue.

The use of PRS in neurosurgery provides considerable advantages over traditional techniques [6]. Specifically, compared with radiosurgery with isotopes or brachytherapy, IR with PRS eliminates inconveniences of isotopes and provides some dosimetric advantages, since adjustable dose rates and steep dose gradients can be obtained by adapting the electron acceleration potential. Additionally, compared to radiosurgical techniques with external beams, the PRS represents a more cost-effective radiosurgical tumor treatment, which can be executed immediately after the stereotactic biopsy without requiring specific radiation-protection measures or dedicated facilities.

On the other hand, the possibility of inducing only selected spherical shaped necrotic areas (Fig. 2b) is one of the major limits of the PRS.

Indeed, tumors are often irregular in shape, consequently, it is necessary to perform a set of treatments of suitable intensity through a predefined path of points of emission. Accuracy in the execution of such treatment (both in terms of the steepness of the dose gradient, and of positioning precision in the points of emission) becomes of paramount importance to keep it minimally invasive, and justifies the development and use of robotic devices. Clearly, the higher the accuracy of the positioning and the ability to drive the PRS during the insertion, the lower the invasiveness of the intervention. The use of robotic systems to position, orientate and guide the PRS and other surgical tools into the brain can obviously guarantee a much higher level of accuracy than classical stereotactic head-frame based techniques (Fig. 3). A possible initial robotic approach 


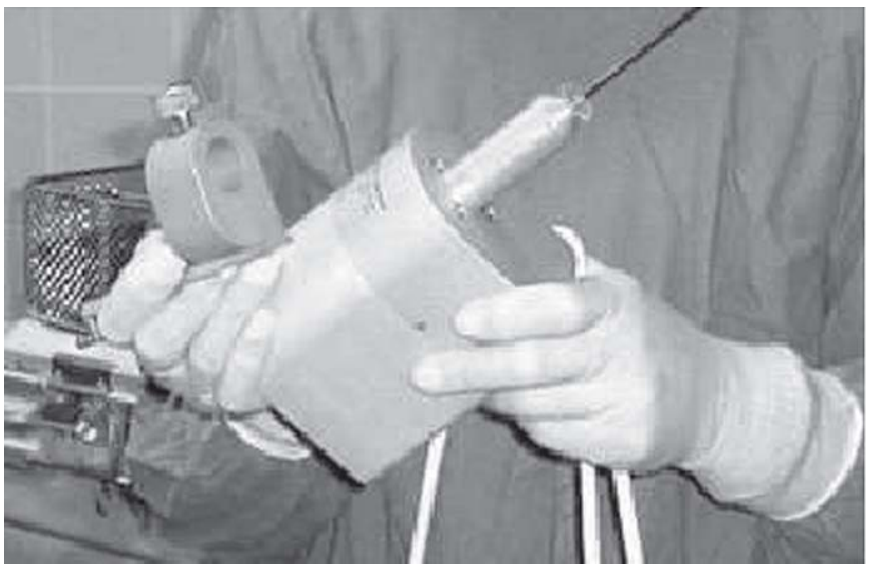

(a)

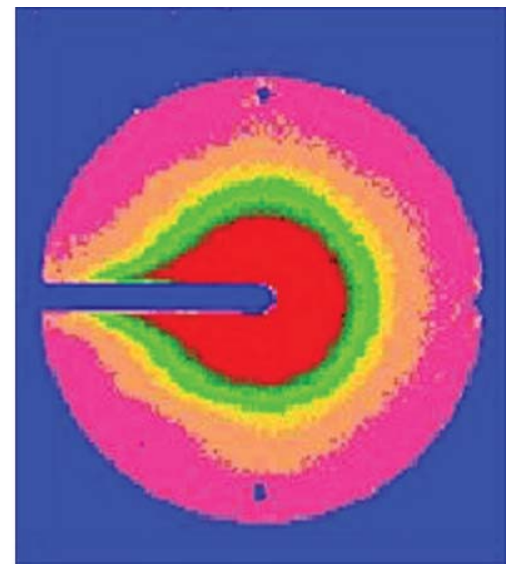

(b)

Fig. 2. PRS Photon Radiosurgery System (by Carl Zeiss ${ }^{\circledR}$. a) The X-ray source (PRS 400) at the Neurosurgical Department, University of Florence, Italy. b) The PRS radiation dose distribution is nearly spherical.

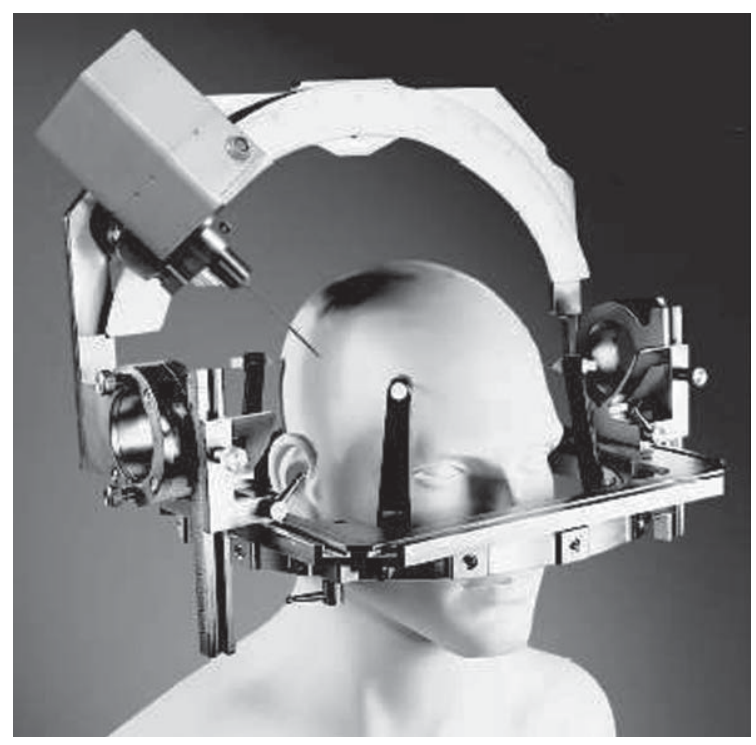

Fig. 3. Stereotactic head-frame.

to the execution of neurosurgical interventions with the PRS might involve the direct use of a commercial surgical robot, as the Neuromate mentioned above. However, Neuromate has some technical limitations [10] which make its use unreliable for the insertion of PRS probes into the brain.

Undoubtedly, precision, accuracy, repeatability and force control are all critical features in surgical procedures, and in particular in PRS IR, not only because erratic motion can be very traumatizing, but also because any deflection of the PRS probe has to be avoided in order to prevent the dissipation of the beam energy and, consequently, a drop in the therapy's beneficial effects. Probe deflections are chiefly caused when the probe makes contact with the skull of the patient. The limited accuracy of the Neuromate [10] and the lack of force control make it impossible to prevent or monitor probe deflections and, hence, ensure the actual minimal invasiveness of procedures.

The above considerations above provide evidence that the robotic systems that are suitable for neurosurgical interventions by PRS, besides being very accurate, must allow real-time measurement of the contact force between the surgical tool and the tissue touched. The contact force has then to be fed back to the surgeon through the interface used to guide the tool's insertion.

This is the robotic configuration adopted in the previous prototype, named LANS Linear Actuator for NeuroSurgery [18]. This system includes a haptic master module (Fig. 4a), operated by the surgeon and a slave module (Fig. 4b), able to move the PRS along a linear trajectory and stop at a position which is dependent on that of the master.

LANS has been designed to be used as the end effector of the Neuromate robot (Fig. 4b).

During a typical procedure, Neuromate is used to bring the LANS slave module close to the insertion point and give the correct orientation to the surgical tool. Following this, the Neuromate's task is over and 


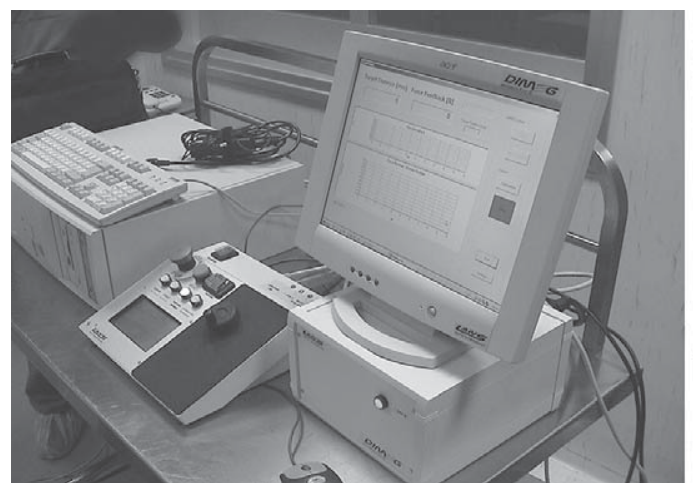

(a)

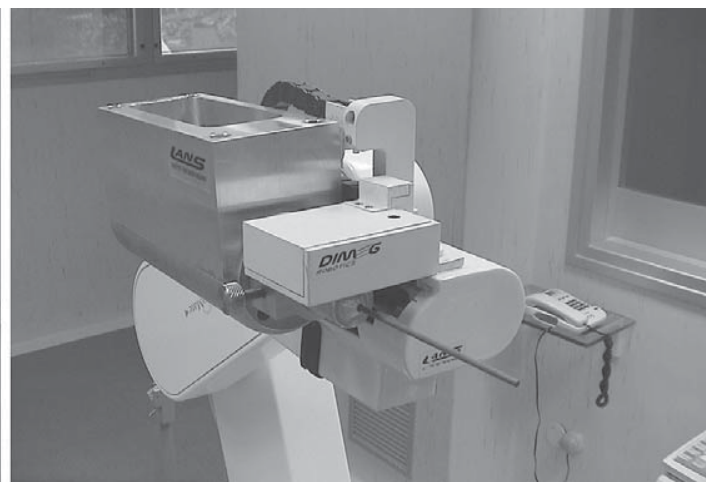

(b)

Fig. 4. Previous prototype: LANS (Linear Actuator for NeuroSurgery). a) Master module and LANS controller. b) Slave module and Neuromate arm at the Neurosurgical Department, University of Florence, Italy.

the insertion of the tool into the brain is entirely executed by LANS.

This procedure allows the motion of the robotic system as a whole to be decoupled into the Neuromate "gross" motion and the LANS "fine" motion, and enhances the system's safety and performance. The high precision ensured by LANS in the positioning of the tool improves the outcome of stereotactic neurosurgical procedures.

As mentioned above, LANS has been designed to perform interventions in tele-operation mode. The surgeon moves the master handle determining the axial position of the PRS, and perceives a force feedback proportional to the contact force between the PRS and the brain. As a result, the surgeon is given the sensation of directly operating on the patient.

\section{DAANS: An improved robot for neurosurgery}

Single PRS radiation produces almost spherical dose distributions (Fig. 2b). As stated above, the challenge is to avoid unacceptable dose delivery to non-target tissue. In recent years, researchers of the Neurosurgical Department of the University of Florence have discovered a way to shape the high-dose region into a variety of profiles that closely conform to the shape of each particular tumour. This can be achieved by using suitable gold-made conformal caps (Fig. 5) and performing multiple-targets dose delivery, as shown in Fig. 6.

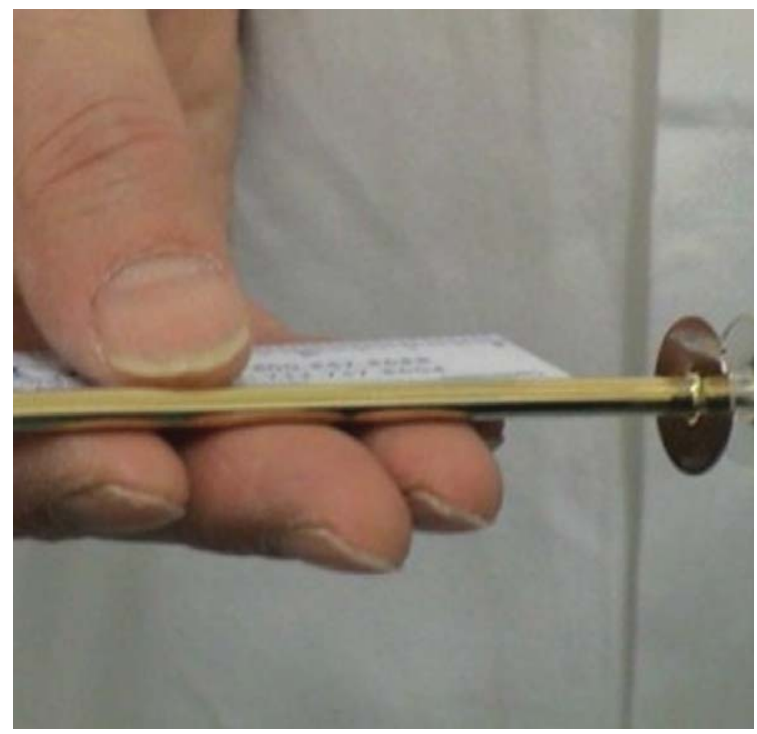

Fig. 5. Gold-made conformal cap used in the improved surgical treatment with PRS.

The conformal caps cover the PRS probe (as shown in Fig. 5) and have small windows on one tip. Gold acts as a shield for X-rays, so that the radiation hits only cerebral tissue beyond the window, while the other regions are protected. This new solution permits the application of the procedure with PRS to lesions of very irregular shape, simply by integrating translation of the source along the emission axis and spinning of the conformal caps around the same axis.

The previous actuator does not allow such an use of the PRS, since only linear motion along the emission axis can be performed. As such, a new actuator has 


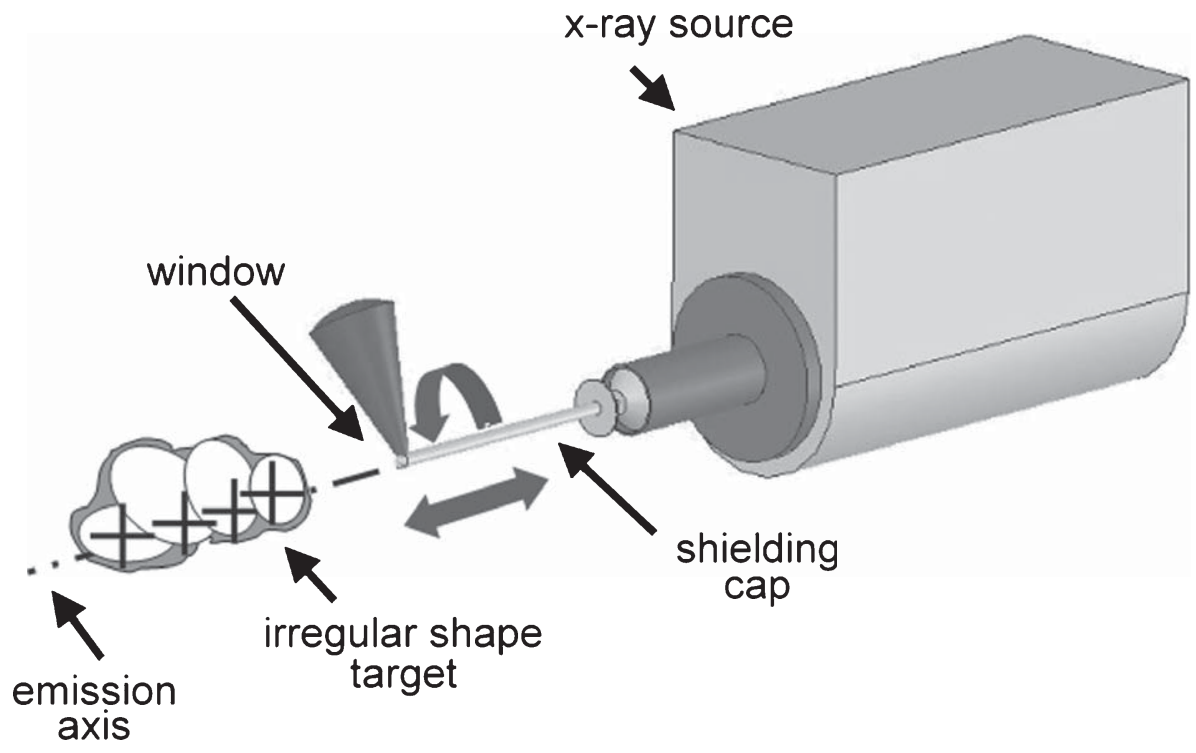

Fig. 6. Innovative treatment with PRS and conformal caps.

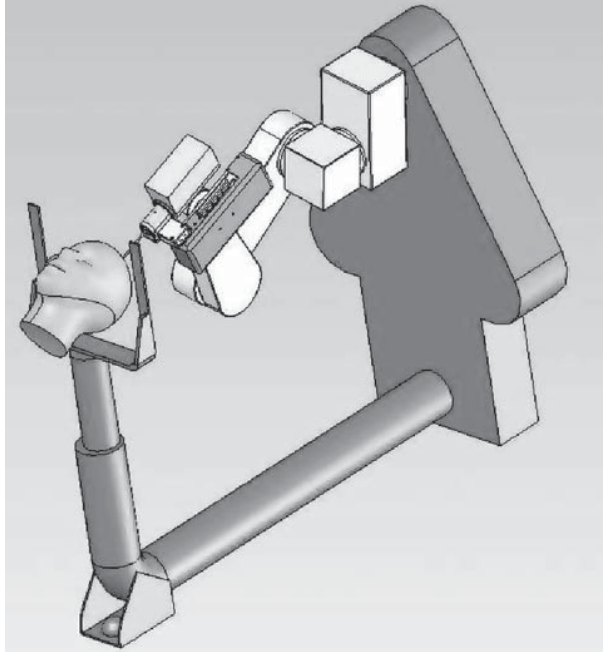

(a)

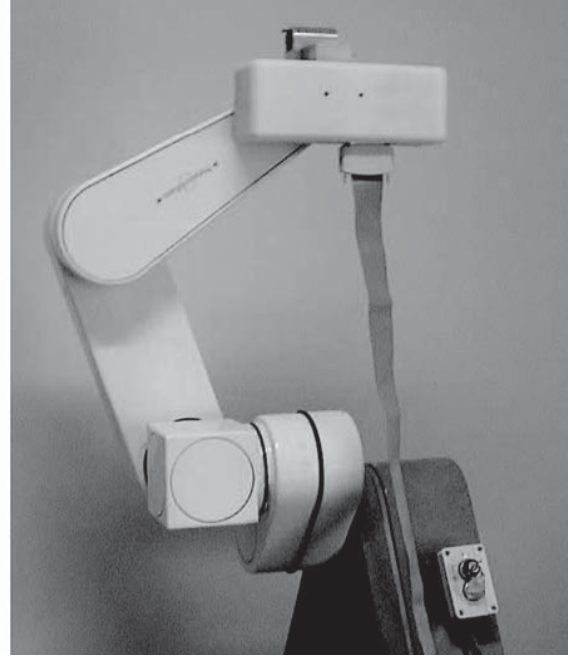

(b)

Fig. 7. DAANS' slave actuator and Neuromate ${ }^{\circledR}$. a) Treatment by DAANS and Neuromate. b) Slave module and Neuromate arm at the Neurosurgical Department, University of Florence, Italy.

been developed to meet the new requirements. The new robotic system is virtually based on the same concept as the previous prototype. However, the whole robotic system had to be re-designed to allow for the rotation of the conformal caps. DAANS has been designed to be used as the end effector of the Neuromate robot. The Neuromate is used to bring the robot close to the insertion point and to give the correct orientation to the surgical tool (Fig. 7a and b).

\subsection{Slave unit}

The slave robot features two degrees of freedom, allowing the linear motion of the PRS along its emis- 


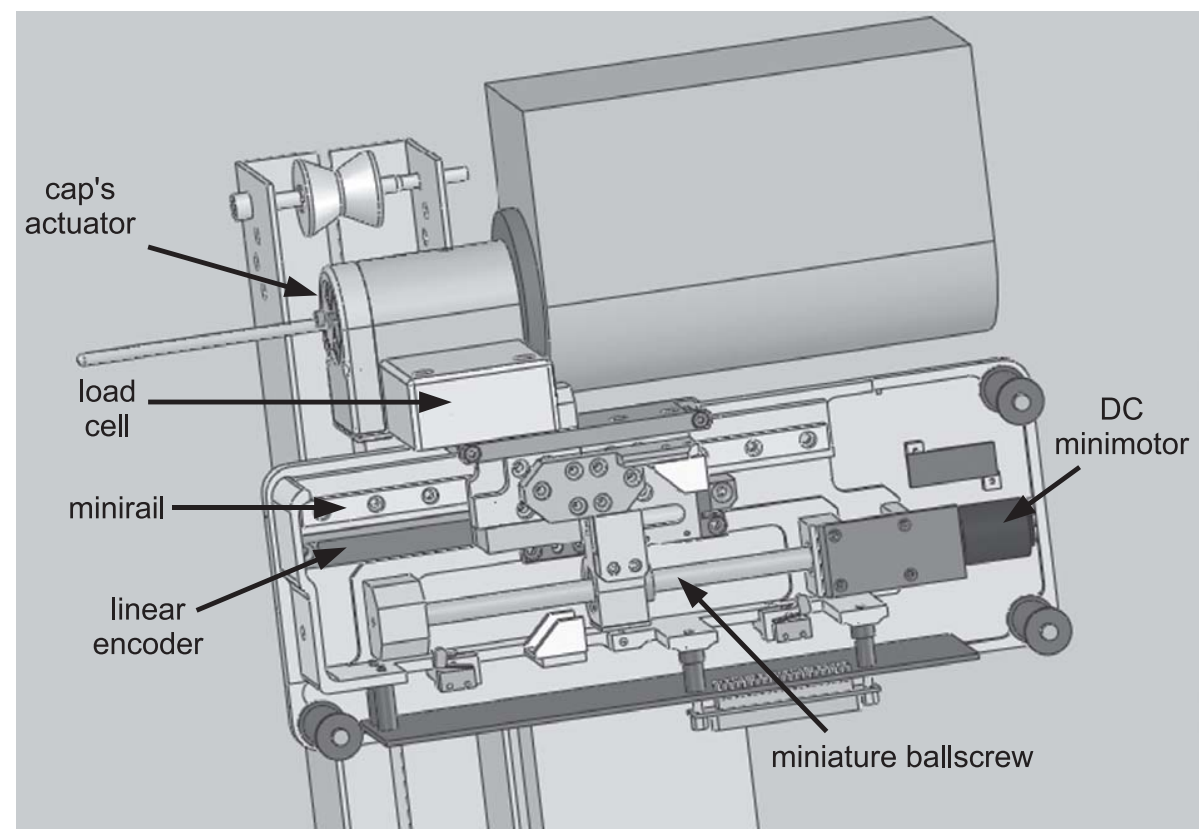

Fig. 8. Slave unit layout.

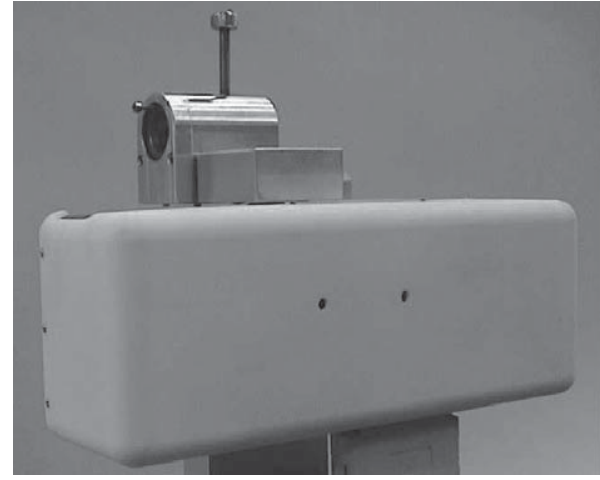

(a)

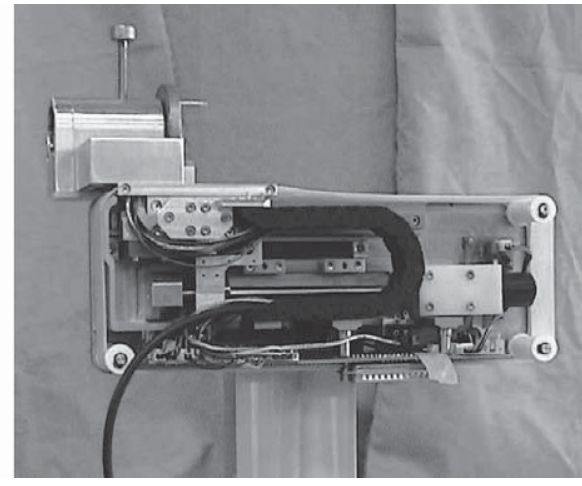

(b)

Fig. 9. DAANS slave unit. a) Slave unit. b) Slave components.

sion axis and the rotation of the conformal caps around the same axis. The actuator for the linear motion (Figs $8,9 \mathrm{a}$ and $\mathrm{b}$ ) is made up of three main parts: a base chassis, which can be easily attached to the Neuromate end-effector, a mobile carriage, and a sensorized tool-holder. A Faulhaber DC micro-motor moves a miniature Steinmeyer ballscrew, whose backlash-free, preloaded single nut shifts the carriage. Motion linearity is nearly $0.01 \mathrm{~mm}$ and is ensured by a Schneeberger Minirail recirculating ball guideway. A load cell is integrated on the support holding the PRS (Fig 10a and b). This device allows the measurements of the contact force between the surgical tools and brain tissue. This signal is read by a computer in order to provide the surgical task performed by the surgeon with a realistic feeling.

A linear encoder joined to the mobile carriage measures the position of the PRS. The accuracy on the linear movement has been experimentally estimated, with results better than $0.025 \mathrm{~mm}$. The main technical characteristics of the linear actuator are summarized in Table 1 . The rotary actuator comprises a frame, connected to the shielding conformal cap, which is moved by a DC micro motor through a geared transmission 


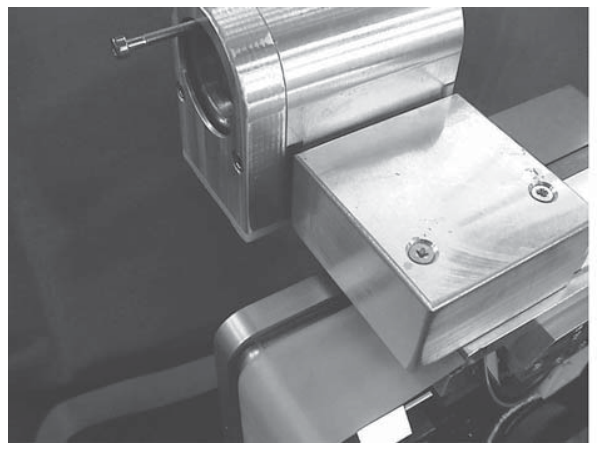

(a)

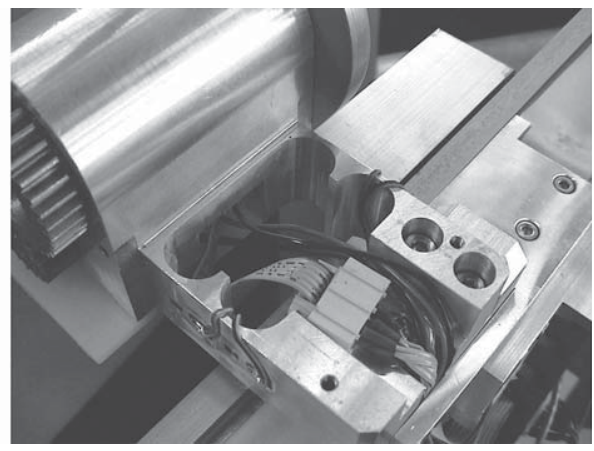

(b)

Fig. 10. Sensorized tool-holder. a) Load cell. b) Load cell layout.

Table 1

Linear actuator components

\begin{tabular}{ll}
\hline DC minimotor & Faulhaber 2224SR \\
Miniature ballscrew & Steinmeyer 1112/0,5.8.145.170.T3P \\
Miniature minirail & Schneeberger MG 9-195-7.5-7.5-G1-V1 \\
Magnetic sensor & SIKO MSK200 5-E1/2,0-I-2-4 \\
Load cell & home-made \\
\hline
\end{tabular}

(Fig. 11a and b). The angular position is measured by means of a rotary encoder mounted on the motor shaft. The main technical characteristics of the conformal cap's actuator are summarized in Tables 2 and 3.

\subsection{Master unit}

The master unit is the device on which the surgeon operates. It is made up of a haptic knob enclosed in a 19' standard rack (Figs 12 and 13a). The knob is a $50 \mathrm{~mm}$ diameter cylinder connected to a torque-controlled DC motor (Fig. 13a). The maximum torque exerted on the surgeon's hand is approximately $140 \mathrm{mNm}$. The knob angular displacement is measured by an incremental encoder mounted on the motor shaft. On the front panel of the chassis, an LCD display, a small keypad, LEDs and push buttons are installed. The LCD display is used to give the operator the most important information about the system, such as the position of the slave and the forces measured. By means of the keypad, it is possible to predispose a change on the surgical tools and set up a new surgical X-ray target. LEDs and push buttons are used to enable the surgeon to react quickly to any dangerous situation. Inside the master chassis, a NI cRIO-9002 embedded computer is installed as the controller for the whole system. Nevertheless, to improve safety during operation, a dedicated FPGA programmable device is used as a redundant control system. If an error is detected, this device stops the operation and assists the surgeon during the emergen-

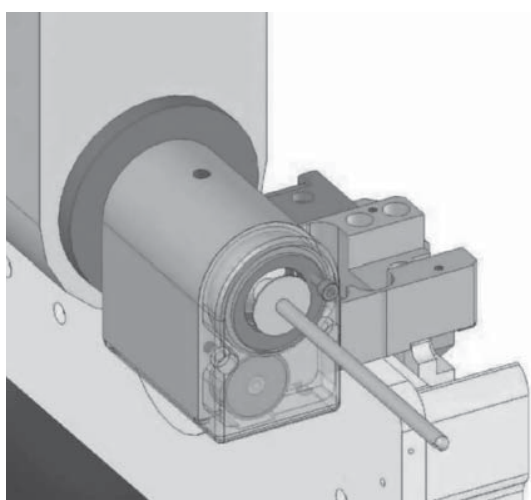

(a)

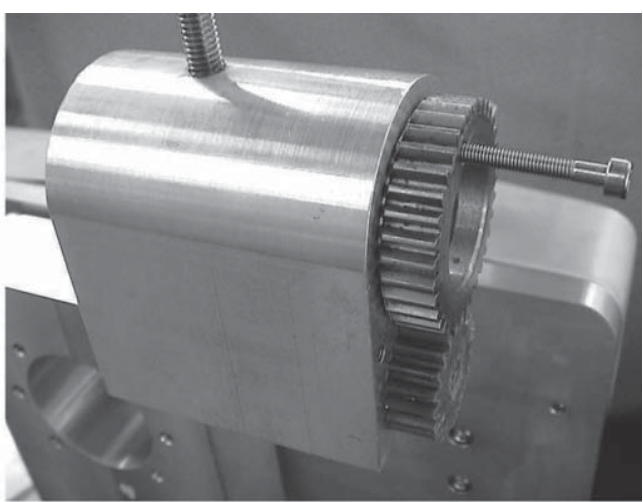

(b)

Fig. 11. Cap's actuator. a) Actuator layout. (b Conformal cap's Actuator. 
Table 2

Conformal caps' actuator components

\begin{tabular}{ll}
\hline DC minimotor & Faulhaber 816P 006S 05AB28 08/1K \\
Magnetic encoder & Faulhaber 05AB28 \\
\hline
\end{tabular}

Table 3

Haptic master components

\begin{tabular}{ll}
\hline DC minimotor & Maxon RE 35 \\
Encoder & Maxon Digital Encoder, Line Drive, Type L \\
\hline
\end{tabular}

cies. The coupling device between the knob and the motor shaft has been designed to allow a one-touch "push-pull lock" (Fig. 13b). This assures quick connection and disconnection of the knob and simplifies the sterilization procedure for the master: only the knob has to be sterilized before a surgical operation, while all other parts of the console can be easily covered with sterilized drapes.

\section{The control system}

By operating the master handle, the surgeon moves the tool actuator and feels the interaction force between the surgical instruments and the cerebral tissue. Therefore, the control system has to manage the signals passing from the environment to the surgeon and vice versa. Moreover, in order to simplify the surgeons' movements during any given procedure and to make the safer, the control has to be able to generate "virtual force". When necessary, surgeon should be prevented from moving the surgical tool freely and dangerously, by making him feel as though the tool were in contact with a "virtual wall" and could not be moved any further. Clearly, meeting all the control requirements defined above makes the design and tuning of the control a very challenging task: it has to both assure robust and stable position control for the slave, as well as force control for the master.

Two master-slave control schemes have to be considered, corresponding to the linear slave movement and the angular conformal cap rotation, respectively. However, they look very similar and can be described by the scheme in Fig. 14. Through a suitable user interface, the surgeon can activate one control system at a time. The same knob generates the signals both for the linear and the rotational movements alternatively.

The square on the top-left side of the scheme represents the surgeon working on the haptic master. The surgeon is described through the sum of two contributions: the operator's impedance which relates the knob velocity to the involuntary operator's force; and an external signal $f_{a}$ which describes the voluntary action of the surgeon on the master. The operator's impedance depends on the characteristic of the surgeon's hand and, for the same operator, is a function of the applied grasp

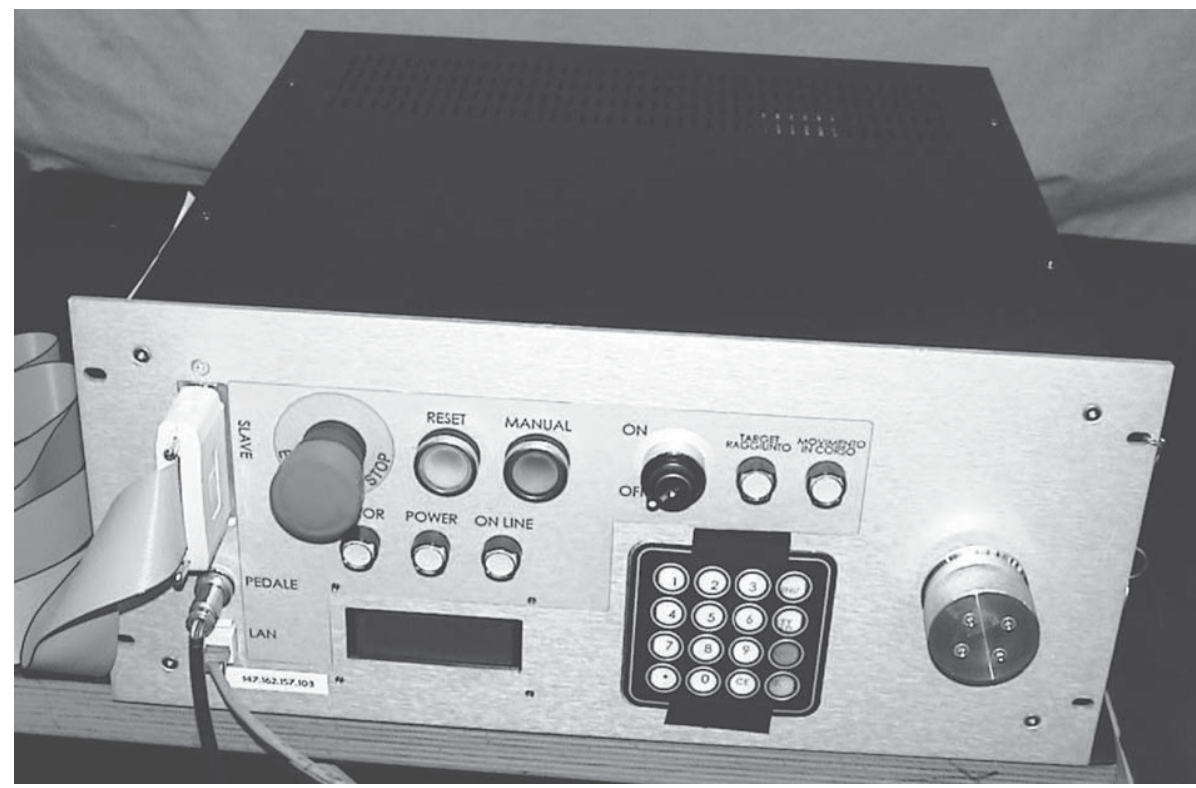

Fig. 12. Master unit of the DAANS 


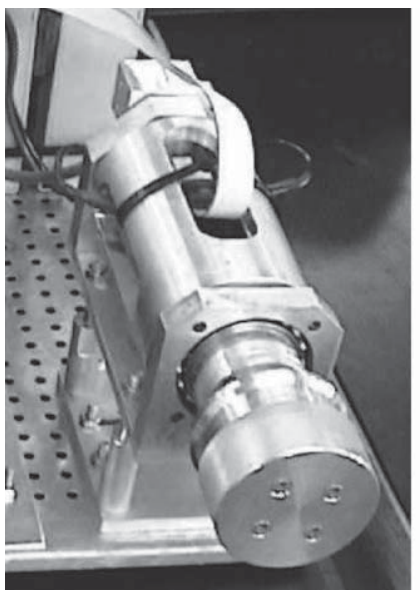

(a)

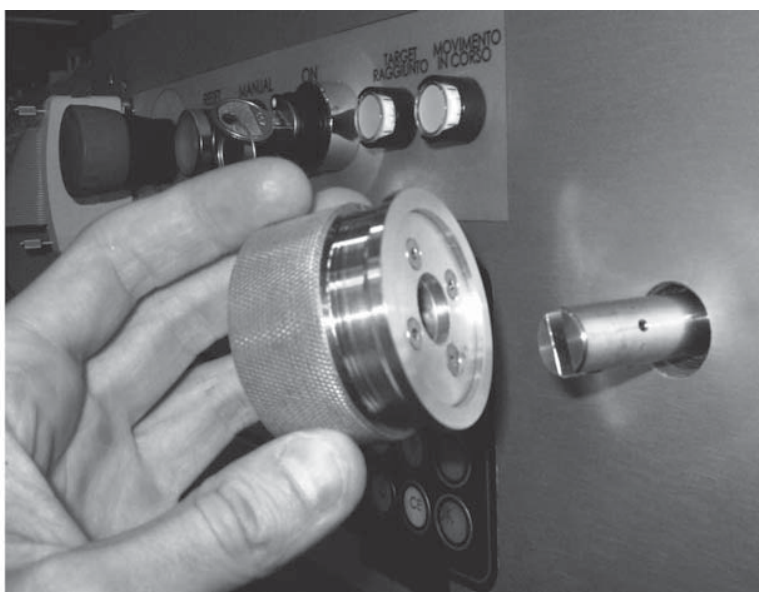

(b)

Fig. 13. Haptic knob. a) Haptic knob and the DC motor. b) Fast removal system for sterilization.

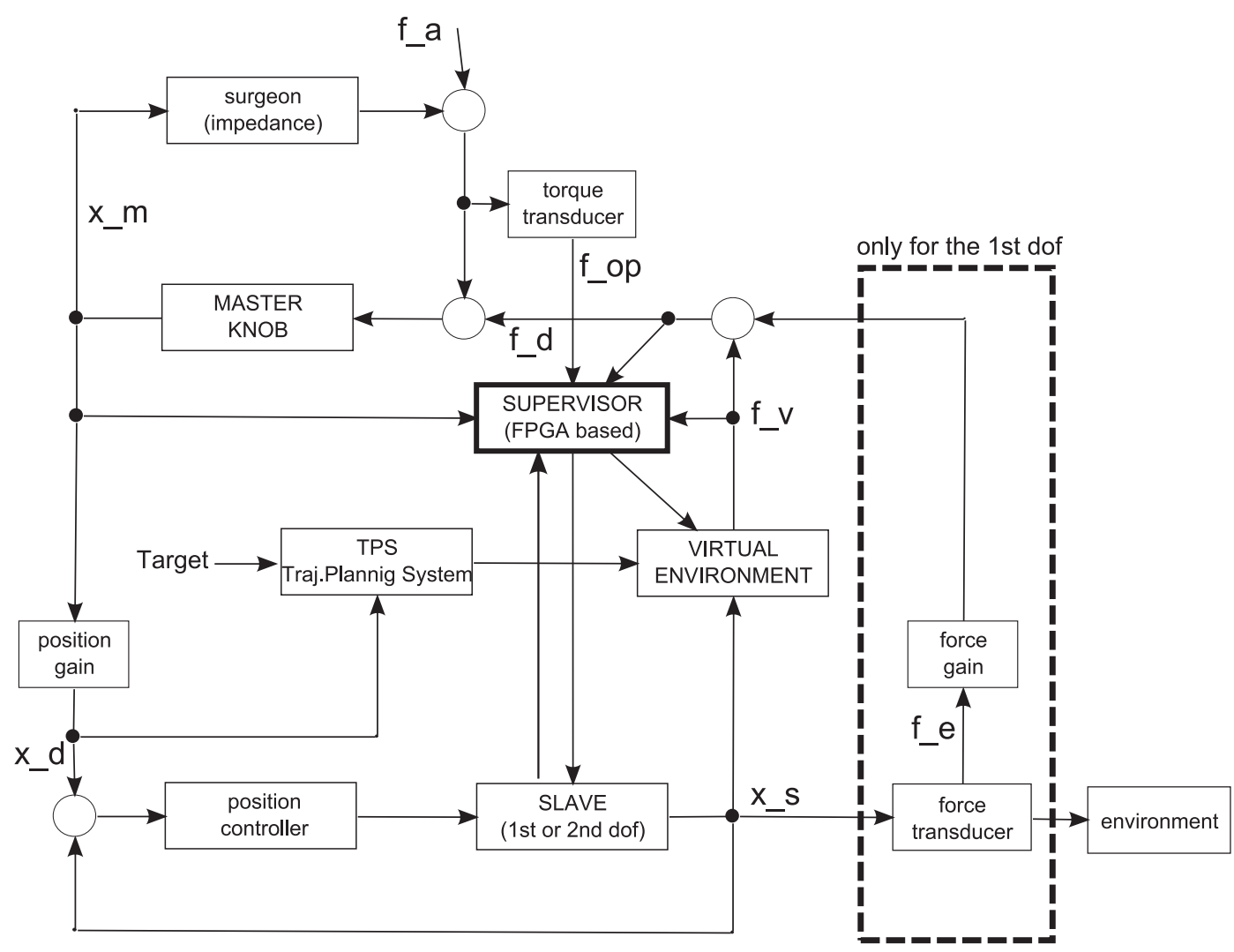

Fig. 14. Control scheme. 
force. Because of this variability, we assume that the impedance is passive. The current position of the knob $\left(x_{m}\right)$ handled by the surgeon produces the reference signal $\left(x_{d}\right)$ for the slave position $\left(x_{s}\right)$. The ratio between $x_{m}$ and $x_{d}$ could be changed for control of the fine movements by changing the position gain. The greater the position gain, the smaller the rotation of the handle necessary to produce the same displacement of the surgical tool. A PID-controlled loop allows the slave to follow the master movements accurately. The load cell placed on the slave unit measures the force between the cerebral tissue and the surgical tools. Subsequently, the controller filters these signals and generates the command signal $f_{f}$ for the torque controlled knob. In this way, the master exerts a torque on the surgeon's hand that follows the current force on the slave, enabling the surgeon to feel what happens between the surgical tool and the cerebral tissue. The ratio between the force measured on the tissue and the torque exerted by the master can be chosen to correspond to the surgeon's sensibility. In order to assure a high level of safety, a Trajectory Planning System (TPS) has been inserted into the scheme, to control the movements of the whole device during each phase of the surgical task. In particular, no slave movements are allowed until a surgical target is defined by the surgeon. Then an operation can start only after the surgeon has pressed a safety footswitch whose function is to enable the slave motors. Moreover, so as to enable movement, which could be very dangerous for the patient, a special "virtual environment" is generated by the TPS, providing the contribute $f_{v}$ with the haptic torque desired. The virtual environment acts as an elastic spring: at the equilibrium point, corresponding to the desired target position, the force signal $f_{v}$ is null. When the surgeon tries to move the master beyond the target (i.e., the equilibrium point for the spring), $f_{v}$ rises and the torque exerted on the surgeon's hand increases proportionally to the distance covered. This behavior forces the master to stay in the correct position. In this manner, the TPS generates a virtual environment able to guide the surgeon during the surgical task. The "supervisor" is a FPGA-based controller which constantly monitors the state of the whole system. It acts as a redundant controller.

The control scheme for the second degree of freedom, which allows the conformal caps to move, looks very similar to the above. However, the reaction torque which occurs during the rotation of the conformal cap is not fed back to the surgeon, since it cannot damage the cerebral tissue.

\section{Conclusion}

In this paper, a new master-slave haptic system for framed stereotactic neurosurgery has been presented. The system, named DAANS (Double Action Actuator for NeuroSurgery), will enable surgeons to move a miniature X-ray source along an arbitrarily chosen emission axis, with a suitable degree of accuracy. Moreover, a haptic console will enable surgeons to feel the contact force between the probe and the cerebral tissue.

The DAANS has been designed for use as the end effector of the Neuromate robot. The Neuromate is employed to bring the robot close to the insertion point and to give the surgical tool the correct orientation. Then, the Neuromate's task is over and the insertion of the tool into the brain is entirely executed by means of the DAANS. This procedure allows the motion of the overall robotic system to be decoupled into the Neuromate "gross" motion and the DAANS "fine" motion, which enhances the system's overall safety and performance. The high precision ensured by the DAANS in tool positioning and the availability of scalable force feedback can considerably reduce invasiveness and improve the outcome of stereotactic neurosurgical procedures which are executed by making use of stereotactic headframes and manual tool insertion mechanisms.

Moreover, with respect to the previous robotic system, named LANS, DAANS features a further degree of freedom which make possible a new radiological treatment that has been discovered by the researchers of the Neurosurgical Department, University of Florence. The suggested new treatment, in addition, greatly extends the minimally invasive radiological treatment to lesions of very irregular shape. This prototype is currently subject to some final improvements.

Clinical evaluation by the staff of the Neurosurgical Clinic of the University of Florence is scheduled for upcoming months. The earliest significant results will be available within a year.

\section{Acknowledgement}

The authors wish to acknowledge the Italian Ministry of Education, University and Research for providing part of the funds necessary for this research. 


\section{References}

[1] A.L. Benabid, P. Cinquin, S. Lavalle, J.F. Le Bas, J. Demongeot and J. de Rougemont, Computer-driven robot for stereotactic surgery connected to CT scan and magnetic resonance imaging. Technological design and preliminary results, Appl Neurophysiol 50 (1987), 153-154.

[2] A.L. Benabid, S. Lavallee, D. Hoffmann, J. Demongeot and F. Danel, Potential use of robots in endoscopic neurosurgery, Acta Neurochir Suppl (Wien) 54 (1992), 93-97.

[3] C.W. Burckhart, P. Flury and D. Glauser, Stereotactic brain surgery, IEEE Engineering in Medicine and Biology 14 (1995), 314-317.

[4] J.M. Drake, M. Joy, A. Goldenberg and D. Kreindler, Computer-and robot assisted resection of thalamic astrocytomas in children, Neurosurgery 29 (1991), 27-31.

[5] R.A. Faust, Robotics in Surgery: History, Current and Future Applications, Nova Science Publishers, New York, 2007.

[6] P. Gallina, C. Cavedon, P. Francescon, F. Casamassima, R. Mungai, P. Perrini, S. Russo and N. Di Lorenzo, Stereotactic interstitial radiosurgery with a miniature X-Ray device in the minimally invasive treatment of selected tumors in the thalamus and the basal ganglia, Stereotact Funct Neurosurg 79 (2002), 202-213.

[7] P. Gallina, P. Francescon, C. Cavedon, F. Casamassima, R. Mungai, G. Terrin, P. Perrini, S. Pallotta, L. Capaccioli and N. Di Lorenzo , Stereotactic interstitial radiosurgery and intraoperative radiotherapy with a miniature $\mathrm{X}$-ray device in the treatment of selected brain tumors. Preliminary results. Radiosurgery. D. Kondiziolka ed., Basel, Karger, 2002, vol 4, pp. $167-178$

[8] R. Garnette, Sutherland, Paul B. McBeth and Deon F. Louw, NeuroArm: an MR compatible robot for microsurgery, International Congress Series 1256 (June 2003), pp. 504-508.

[9] T. Haidegger, L. Kovacs, G Fordos, Z. Benyo and P. Kazanzides, Future Trends in Robotic Neurosurgery. Proc of 14th Nordic-Baltic Conf on Biomedical Engineering and Medical Physics, 2008

[10] T. Haidegger and P. Tian Xia Kazanzides, Accuracy improvement of a neurosurgical robot system, In Proc of BioRob 2008. 2nd IEEE RAS and EMBS Int Conf on Biomedical Robotics and Biomechatronics, 19-22 Oct. 2008, pp. 836-841.

[11] Kazuhiro Hongo, Tetsuya Goto, Yukinari Kakizawa, Jun-ichi Koyama, Toshikazu Kawai, Kazutoshi Kan, Yuichiro Tanaka and Shigeaki Kobayashi, Micromanipulator system (NeuRobot): Clinical Application in Neurosurgery International Congress Series, Vol. 1256, June 2003, pp. 509-513.

[12] Y.S. Kwoh, J. Hou, E.A. Jonckheere and S. Hayati, A previous termrobotnext term with improved absolute positioning accuracy for CT guided stereotactic brain previous termsurgery, IEEE Trans Biomed Eng 35 (1988), 153-160.

[13] P. McBeth, M.D. Deon F. Louw, Peter R. Rizun B.A.Sc.a and M.D. Garnette R. Sutherland, Robotics in neurosurgery, The American Journal of Surgery 188(4) Supplement 1 (October 2004), 68-75.

[14] T. Miyahara, T. Goto, Y. Kakizawa, J. Koyama, Y. Tanaka and K. Hongo, Neurosurgical telecontrolled micromanipulator system (NeuRobot): Clinically applied cases and further development, International Congress Series 1281 (May 2005), 13-63.

[15] V. Modrak, J. Pako and S. Pavlenko, Alternative solution for a robotic stereotactic system, Journal of Intelligent and Robotic Systems 35(2) (October 2002), 193-202(10).

[16] N. Nathoo, M.C. Cavusoglu, M.A. Vogelbaum and G.H Barnett, In touch with robotics: neurosurgery for the future Neurosurgery 56(3) (Mar. 2005), 421-433.

[17] G. Pantazis, M. Trippel, W. Birg, C. Ostertag and G. Nikkhah, Stereotactic interstitial radiosurgery with the photon radiosurgery system (PRS) for metastatic brain tumors: a prospective single-center clinical trial, International Journal of Radiation Oncology*Biology*Physics, 75(5) (1 December 2009), 1392-1400.

[18] A. Rossi, A. Trevisani and V. Zanotto, A telerobotic haptic system for minimally invasive stereotactic neurosurgery, The International Journal of Medical Robotics and Computer Assisted Surgery 1(2) (2005), 64-75.

[19] L. Zamorano, Q. Li, S. Jain and G. Kaur, Robotics in neurosurgery: state of the art and future technological challenges, Int J Med Robot 1(1) (June 2004), 7-22.

[20] Zengmin Tian, Junchuan Liu, Yuru Zhang, Tianmiao Wang and Hongguang Xing, NeuroMaster: A Robot System for Neurosurgery, IEEE International Conference on Robotics and Automation, 2004. Proceedings. ICRA '04. 2004.

[21] M. Zimmermann, R. Krishnan, A. Raabe and V. Seifert, Robot-assisted navigated neuroendoscopy, Neurosurgery 51(6) (December 2002), 1446-1452. 

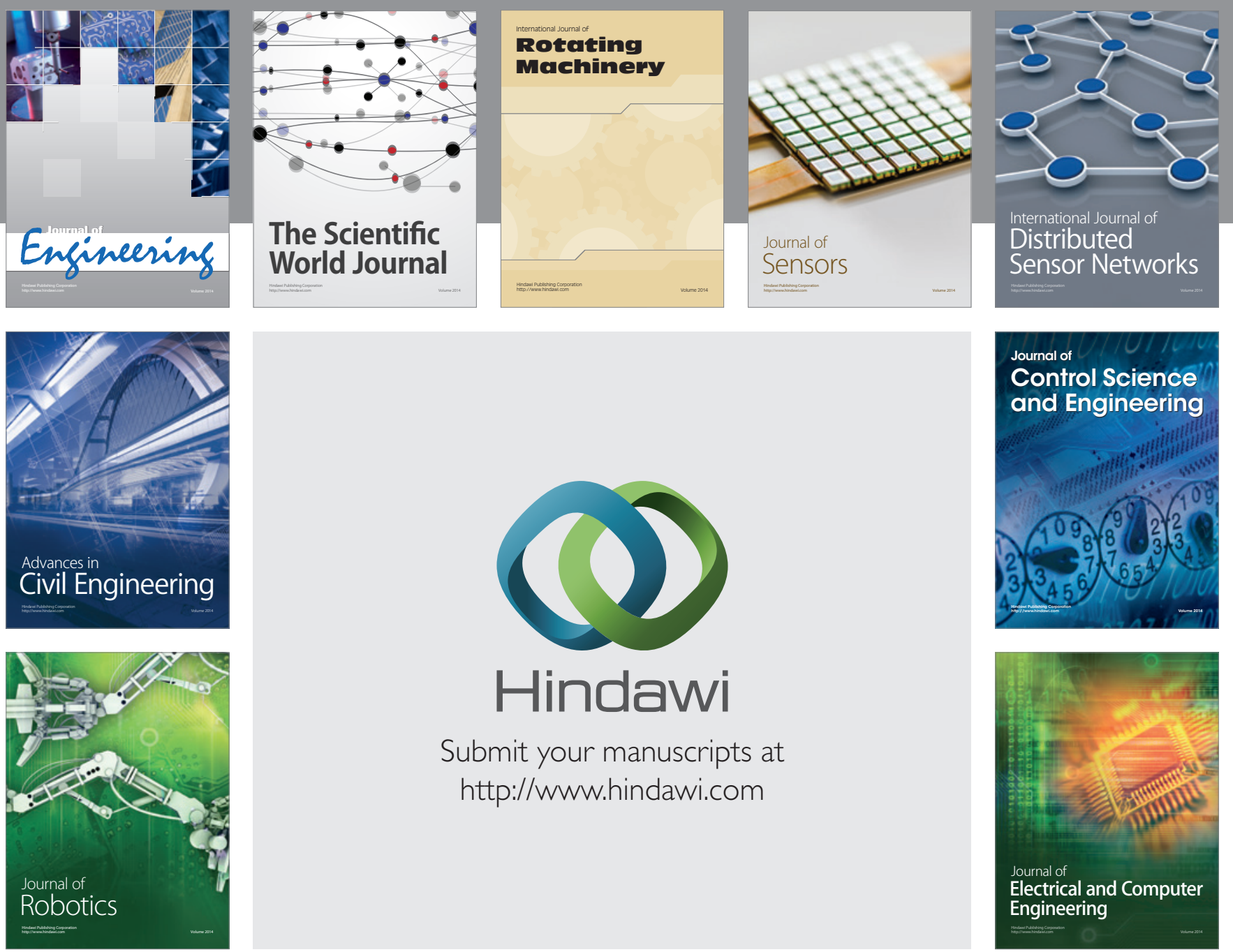

Submit your manuscripts at

http://www.hindawi.com
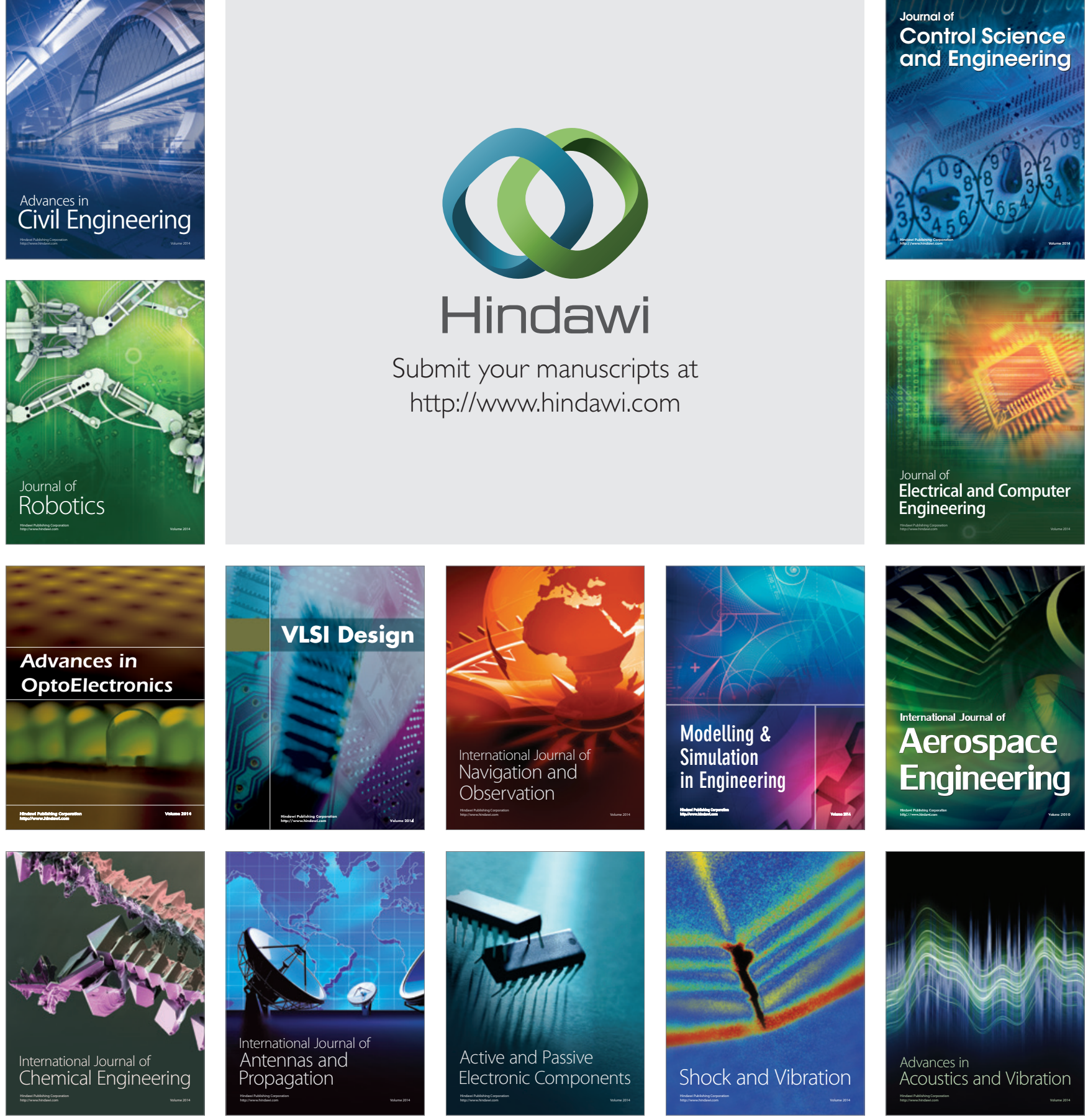